\title{
Identification of DQ Serpentis and DT Serpentis as symbiotic stars ${ }^{\star}$
}

\author{
D. Cieslinski ${ }^{1}$, J.E. Steiner ${ }^{2}$, F. Elizalde ${ }^{1}$, and M.G. Pereira ${ }^{1}$ \\ 1 Divisão de Astrofísica, Instituto Nacional de Pesquisas Espaciais, CP 515, 12201-970 São José dos Campos, Brazil \\ 2 Instituto Astronômico e Geofísico, Universidade de São Paulo, CP 9638, 01065-970 São Paulo, Brazil
}

Received June 11; accepted October 18, 1996

\begin{abstract}
Optical and near infrared spectroscopy of the stars DQ Ser and DT Ser are presented. DQ Ser shows a late-type continuum (M3 - M5) with emission lines of Balmer series, HeI, HeII, [OIII], OI and CaII triplet while DT Ser presents a earlier spectral type with emission of Balmer lines, HeI, HeII and [OIII]. DT Ser is a faint $V=$ 15.4 star 5 arcsec from a $V=12.8 \mathrm{G}$ companion. We suggest it to be a yellow symbiotic star.
\end{abstract}

Key words: stars: binaries: symbiotic - stars: emission line - stars: DQ Ser, DT Ser

\section{Introduction}

Symbiotic stars are long orbital period interacting binaries consisting of a late-type $(\mathrm{G}-\mathrm{M})$ giant which sheds material via stellar wind or Roche lobe overflow onto a more compact and hotter companion. The hot star provides an intense ultraviolet radiation field which ionizes a portion of the surrounding nebula, giving rise to emission lines like HI, HeI, HeII and [OIII] seen in these variables, while characteristics like strong red continuum, $\mathrm{TiO}$ and $\mathrm{VO}$ absorption bands, absorption lines of neutral and singly ionized metals are associated with the late-type star. The compact object in symbiotic stars is in most cases a white dwarf, which can derive its high luminosity and temperature by releases of potential energy in an accretion disk or as result of burning of hydrogen being accreted by the white dwarf or even from the cooling of an object resembling a nucleus of a planetary nebula. Low mass main sequence stars with an accretion disk can also occur as the ionizing source in such systems. For more details about the symbiotic stars see, e.g., Friedjung \& Viotti (1982); Kenyon (1986) and Mikołajewska et al. (1988).

Send offprint requests to: D. Cieslinski, deo@das.inpe.br

* Based on observations made at Laboratório Nacional de Astrofísica/CNPq, Brazil.
Table 1. Log of the spectroscopic observations

\begin{tabular}{llllll}
\hline Name & Date & $\begin{array}{l}\text { spectral } \\
\text { range }\end{array}$ & S/N & $\begin{array}{l}\text { slit } \\
\left({ }^{\prime \prime}\right)\end{array}$ & $\#^{\mathrm{b}}$ \\
\hline DQ Ser & 18 Aug. (1995) & $5960-7260$ & $19^{\mathrm{c}}$ & 3.1 & $\mathrm{~b}$ \\
& 01 Sep. (1995) & $6040-10200$ & 32 & 4.0 & $\mathrm{~d}$ \\
& 05 Sep. (1995) & $4120-5530$ & 7 & 3.1 & $\mathrm{a}$ \\
& 04 Oct. (1995) & $3420-8130$ & 24 & 2.7 & $\mathrm{c}$ \\
DT Ser & 18 Aug. (1995) & $5960-7260$ & $14^{\mathrm{d}}$ & 3.1 & \\
& 02 Sep. (1995) & $6040-10200$ & $6^{\mathrm{d}}$ & 3.1 & $\mathrm{f}$ \\
& 05 Sep. (1995) & $4120-5530$ & $10^{\mathrm{d}}$ & 3.1 & $\mathrm{e}$ \\
& 04 Oct. (1995) & $3420-8130$ & $18^{\mathrm{d}}$ & 2.7 & \\
\hline
\end{tabular}

a) measured on the extracted spectrum over a $\Delta \lambda=100 \AA$ region centered close to the mid position of the observed spectral range,

b) spectra shown in Fig. 1,

c) contaminated by emission lines from the star,

d) contaminated by the bright companion star.

In a previous paper (Cieslinski et al. 1994) we presented observations of 6 symbiotic star candidates. In this paper we describe observations of two additional variables that we suggest as newly identified symbiotic stars. They show emission lines of HI, [OIII] and HeII $\lambda 4686$, characteristics of this class of variables.

\section{Observations and data reduction}

The observations were carried out using the facilities of Laboratório Nacional de Astrofísica (LNA/CNPq), in southeast Brazil. The spectroscopic observations (Table 1) were obtained with a Boller \& Chivens Cassegrain spectrograph attached to the 1.6-m telescope. Two gratings with 300 and 900 lines $/ \mathrm{mm}$ were used, which provide about $10 \AA$ and $3.5 \AA$ resolution (as derived from the FWHM of the lines from comparison lamps). The detector used was 

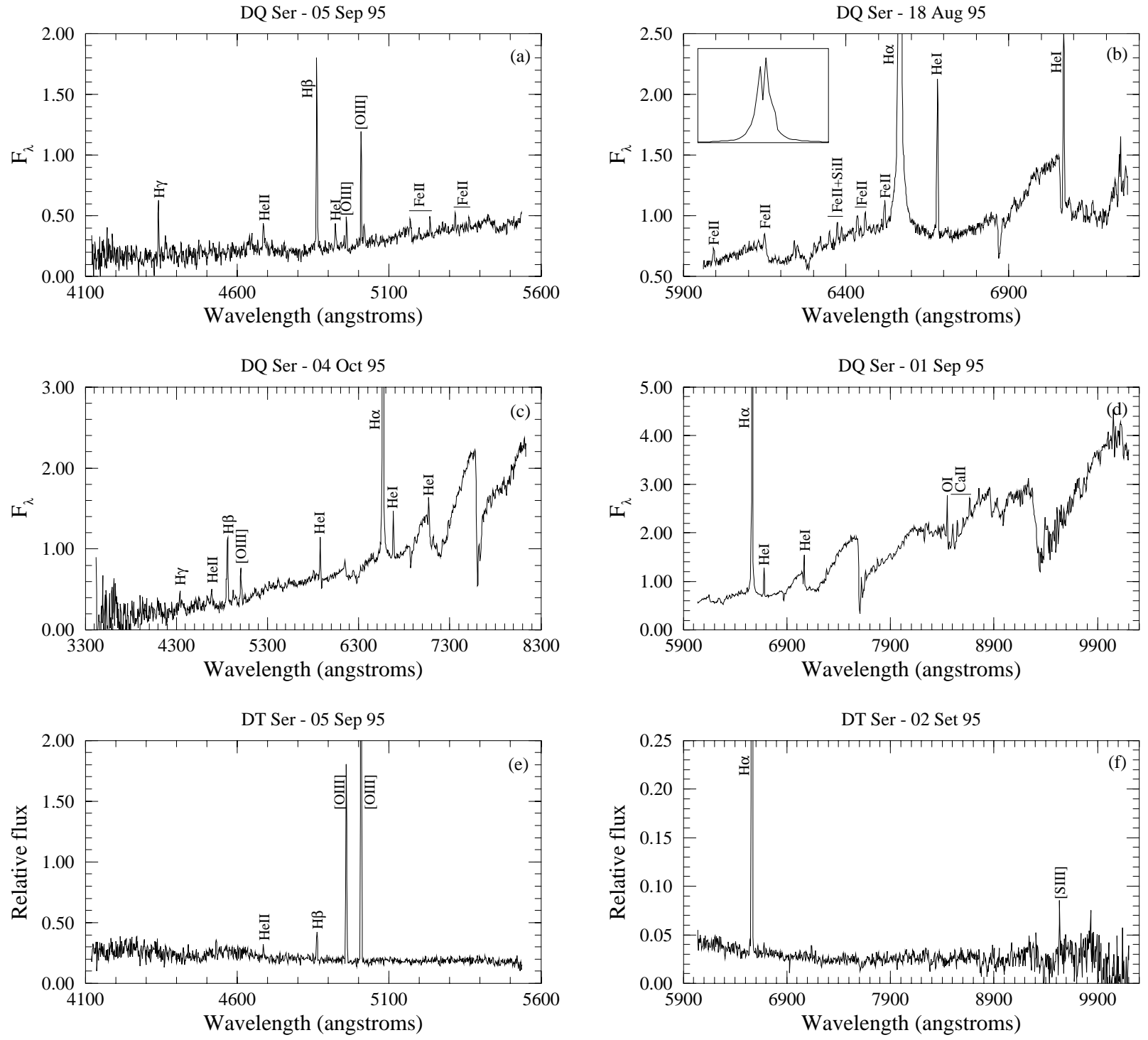

Fig. 1. Spectra of DQ Ser $(\mathbf{a}, \mathbf{b}, \mathbf{c}, \mathbf{d})$ and DT Ser $(\mathbf{e}, \mathbf{f})$. The fluxes are in units of $10^{-14} \mathrm{erg} \mathrm{cm}^{-2} \mathrm{~s}^{-1} \AA^{-1}$

Table 2. $U B V(R I)_{\mathrm{C}}$ photometry

\begin{tabular}{lcccccccccc}
\hline JD & $V$ & $U-B$ & $B-V$ & $V-R_{\mathrm{C}}$ & $R_{\mathrm{C}}-I_{\mathrm{C}}$ & $\sigma_{V}$ & $\sigma_{U-B}$ & $\sigma_{B-V}$ & $\sigma_{V-R}$ & $\sigma_{R-I}$ \\
\hline DQ Ser & & & & & & & & & & \\
2448830.6577 & 14.65 & 0.05 & 1.22 & 1.30 & 1.39 & 0.04 & 0.15 & 0.07 & 0.04 & 0.02 \\
2449953.4683 & 14.45 & 0.28 & 1.25 & 1.31 & 1.46 & 0.04 & 0.19 & 0.08 & 0.04 & 0.03 \\
DT Ser & & & & & & & & & & \\
2450250.62666 & 15.40 & -0.59 & 0.51 & -0.06 & 0.10 & 0.05 & 0.07 & 0.07 & 0.06 & 0.07 \\
\hline
\end{tabular}


a GEC P88230 CCD $(770 \times 1152$ pixels, $22.5 \mu \mathrm{m} /$ pixel $)$ with UV coating. In the near infrared we used a OG550 filter in order to cut the blue portion of the second order spectrum.

The spectra were extracted using the Image Reduction and Analysis Facility (IRAF ${ }^{1}$ ) software installed at the SUN workstations of the Astrophysics Division at INPE. Spectrophotometric standard stars from the lists of Stone \& Baldwin (1983) and Taylor (1984) were observed each night to flux calibrate the spectra. The integrated line fluxes of DQ Ser were computed using the "splot" task of IRAF, and are presented in Table 3 . The line fluxes were not corrected for reddening and we estimate the absolute fluxes to be accurate to about $30 \%$, while relative line fluxes being more accurate.

The $U B V R I$ data were collected using the FOTRAP photometer at the 0.6-m Zeiss and 1.6-m Boller \& Chivens telescopes. This photometer uses a high-speed rotating (1200 rpm) filter wheel which allows quasi-simultaneous photometry in 5 bands. The detector used was a thermoelectrically cooled Hamamatsu R943-02 photomultiplier operated in pulse counting mode.

The instrumental magnitudes were transformed to the $U B V(R I)_{\mathrm{C}}$ system by observing standard stars in the Eregions (Graham 1982). The data obtained are shown in Table 2. The uncertainties (Cols. 7 to 11) take into account the errors due to photon noise in the measurements and errors associated with the transformation to the standard system. A more complete description of the procedures of reduction, as well of the FOTRAP photometer and the photometric system, can be found in Jablonski et al. (1994).

\section{Discussion}

\subsection{DQ Serpentis}

The star DQ Ser $(l=36.7 ; b=3.8)$ is quoted in the 4th ed. of General Catalogue of Variable Stars-GCVS4 (Kholopov et al. 1987) as a slow irregular variable (type L) presenting a brightness variation from 13.9 to $16.0 \mathrm{mag}$. Our spectra show a late continuum with emission lines of Balmer series, HeI $(\lambda \lambda 5876,6678,7065)$, HeII $\lambda 4686$, CIIINIII $\lambda 4640-4650$, [OIII] $(\lambda \lambda 4363,4959,5007)$, OI $\lambda 8446$ and CaII triplet $(\lambda \lambda 8498,8542,8662)$. Lines of Paschen series in $8598 \AA$ and $8751 \AA$, as well a large number of FeII lines are also seen (Figs. 1a, b, c, d). The continuum of the late-type star is compatible with a type M3-M5 when compared with the spectra presented in Jacoby et al. (1984), Schulte-Ladbeck (1988) and Medina Tanco \& Steiner (1995). The presence of a late-type con-

1 IRAF is distributed by National Optical Astronomy Observatories, which is operated by the Association of Universities for Research in Astronomy, Inc., under contract with the National Science Foundation.
Table 3. Observed lines fluxes of DQ Ser scaled to $\mathrm{H} \beta=1.00$ or $\mathrm{H} \alpha=1.00$

\begin{tabular}{lrrrr}
\hline Line & \multicolumn{5}{c}{ DQ Ser } \\
\hline $\mathrm{H} \gamma \lambda 4340$ & 0.28 & 0.018 & - & - \\
{$[\mathrm{OIII}] \lambda 4363$} & 0.15 & 0.012 & - & - \\
$\mathrm{HeII} \lambda 4686$ & 0.22 & 0.029 & - & - \\
$\mathrm{H} \beta \lambda 4861$ & 1.00 & 0.12 & - & - \\
{$[\mathrm{OIII}] \lambda 4959$} & 0.14 & 0.021 & - & - \\
{$[\mathrm{OIII}] \lambda 5007$} & 0.47 & 0.054 & - & - \\
$\mathrm{HeI} \lambda 5876$ & - & 0.043 & - & - \\
$\mathrm{H} \alpha \lambda 6563$ & - & 1.00 & 1.00 & 1.00 \\
$\mathrm{HeI} \lambda 6678$ & - & 0.054 & 0.060 & 0.062 \\
$\mathrm{HeI} \lambda 7065$ & - & 0.036 & 0.054 & 0.057 \\
OI $\lambda 8446$ & - & - & 0.083 & - \\
CaII $\lambda 8498+\mathrm{HI}(\mathrm{P} 16)$ & - & - & 0.086 & - \\
CaII $\lambda 8542+\mathrm{HI}(\mathrm{P} 15)$ & - & - & 0.037 & - \\
CaII $\lambda 8662+\mathrm{HI}(\mathrm{P} 13)$ & - & - & 0.041 & - \\
$-\log F(\mathrm{H} \beta)\left(\mathrm{erg} \mathrm{s}^{-1} \mathrm{~cm}^{-2}\right)$ & 13.23 & 12.82 & - & - \\
$-\log F(\mathrm{H} \alpha)\left(\mathrm{erg} \mathrm{s}^{-1} \mathrm{~cm}^{-2}\right)$ & - & 11.90 & 12.05 & 11.97 \\
Spectrum label & $(\mathrm{a})$ & $(\mathrm{c})$ & $(\mathrm{d})$ & $(\mathrm{b})$ \\
\hline
\end{tabular}

a: see Table 1

- : lines out of the spectrum range.

tinuum with emission lines of HI, [OIII] and HeII indicates DQ Ser as a symbiotic star.

The $U-B$ and $B-V$ colours presented by DQ Ser (Table 2) are somewhat bluer than those expected from a M3-M5 giant without emission lines. This is characteristic of symbiotic stars in which $\mathrm{H} \beta+[\mathrm{OIII}]$ lines enhance the emission in the filter $B$ while the Balmer jump in emission, quite common in such variables, can affect the $U-B$ colour by a significant amount (Munari et al. 1992; Kenyon 1986). The $V-I$ index which is not strongly affected by emission lines is also consistent with symbiotic M giants (Munari et al. 1992).

DQ Ser also shows a double and asymmetric profile in the $\mathrm{H} \alpha$ line, whose peaks are centred in 6563.9 $\AA$ and in $6566.2 \AA$ (see inserted box in Fig. 1b). Such $\mathrm{H} \alpha$ profiles are quite common among symbiotic stars (Van Winckel et al. 1993).

\subsection{DT Serpentis}

DT Ser $\left(l=25.9 ; b=10^{\circ} 3\right)$ appears in the GCVS4 as a probable irregular variable of type I, with a brightness variation in the range $13.2-13.9 \mathrm{mag}$. Due to its spectral type G0 with lines of [OIII] $(\lambda \lambda 4959,5007)$, Bond (1978) suggested a classification as probable symbiotic.

Accurate measurements of DT Ser are difficult because of its close and bright companion star. This star, about 5 arcsec from the variable, has $V$ magnitude of 12.8 and $B-V$ of 0.76 . Our spectra of this companion 

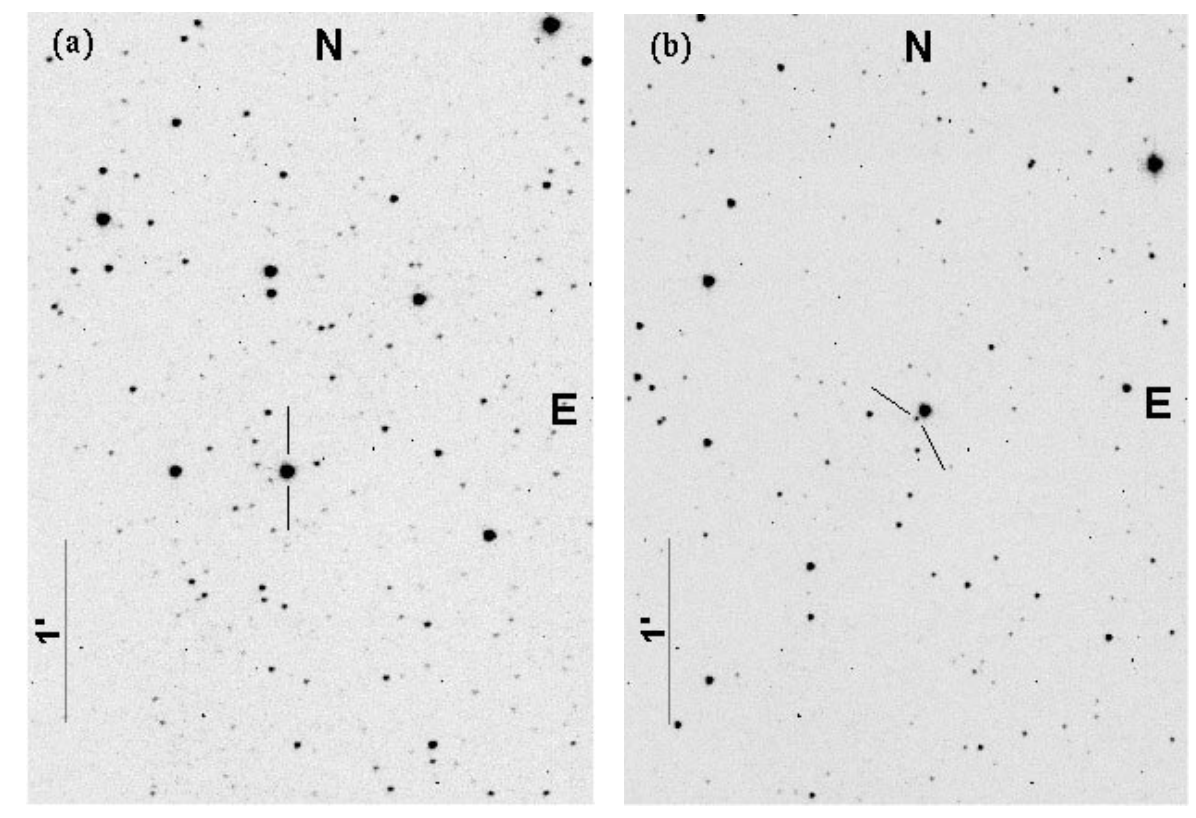

Fig. 2. Finding chart for DQ Ser a) and DT Ser b). These images were obtained in the $I$ filter and cover about $3.0 \times 4.2$ arcmin. North is up and east is right

show the Balmer series in absorption. Other absorption lines, including the $G$ band, are also present. DT Ser is $\sim 2.6$ mags fainter as estimated from our $U B V R I$ photometry (Table 2). The extracted spectrum of the variable shows Balmer lines in emission as well as [OIII] $(\lambda \lambda 5007$, $4959)$ and $\mathrm{HeI} \lambda 5876$. A weak emission of HeII $\lambda 4686$ is also present (Figs. 1e, f). The continuum is inconsistent with being a $\mathrm{M}$ or $\mathrm{K}$ giant as it does not display any band associated with these spectral types. We suggest this star to be a yellow symbiotic. We should, however, note that it is difficult to assign a specific spectral type because of the light contamination from bright $\mathrm{G}$ companion. This fact also makes the measurements of line fluxes and line ratios not reliable; so we do not included them in Table 3.

Figures $2 \mathrm{a}$ and $\mathrm{b}$ show finding charts for these objects.

Acknowledgements. We would like to thank R. Baptista, F.J. Jablonski and G.R. Hickel for obtaining UBVRI photometry. We also thank the referee, R. Viotti, for his valuable comments and suggestions. D. Cieslinski and F. Elizalde thank for the support of CNPq (Conselho Nacional de Desenvolvimento Científico e Tecnológico), under contracts 140587/89-6 and 142589/92-6.

\section{References}

Bond H.E., 1978, PASP 90, 526

Cieslinski D., Elizalde F., Steiner J.E., 1994, A\&AS 106, 243

Friedjung M., Viotti R., 1982, in: The nature of symbiotic stars, IAU Coll. 70. Reidel, Dordrecht

Graham J.A., 1982, PASP 94, 244

Jablonski F., Baptista R., Barroso Jr.J., et al., 1994, PASP 106,1172

Jacoby G.H., Hunter D.A., Christian C.A., 1984, ApJS 56, 257

Kenyon S.J., 1986, The Symbiotic Stars. Cambridge University Press, Cambridge

Kholopov P.N., et al., 1987, General Catalogue of Variable Stars-GCVS4. Nauka Publishing House, Moscow

Medina Tanco G.A., Steiner J.E., 1995, AJ 109, 1770

Mikołajewska J., Friedjung M., Kenyon S.J., Viotti R., 1988, in: The symbiotic phenomenon, IAU Coll. 103. Kluwer Academic Publishers, Dordrecht

Munari U., Yudin B.F., Taranova O.G., et al., 1992, A\&AS 93, 383

Schulte-Ladbeck R.E., 1988, A\&A 189, 97

Stone R.P.S., Baldwin J.A., 1983, MNRAS 204, 347

Taylor B.J., 1984, ApJS 54, 259

Van Winckel H., Duerbeck H.W., Schwarz H.E., 1993, A\&AS 102,401 